\title{
A gauge invariant discretization on simplicial grids of the Schrödinger eigenvalue problem in an electromagnetic field
}

\author{
Snorre Harald Christiansen* and Tore Gunnar Halvorsen ${ }^{\dagger}$ \\ Centre of Mathematics for Applications, University of Oslo, \\ P.O. Box 1053 Blindern, 0316 Oslo, Norway
}

April 16, 2009

\begin{abstract}
We propose a method to compute approximate eigenpairs of the Schrödinger operator on a bounded domain in the presence of an electromagnetic field. The method is formulated for the simplicial grids that satisfy the discrete maximum principle. It combines techniques from lattice gauge theory and finite element methods, retaining the discrete gauge invariance of the former but allowing for non-congruent space elements as in the latter. The error in the method is studied in the framework of Strang's variational crimes, comparing with a standard Galerkin approach. For a smooth electromagnetic field the crime is of order the mesh width $h$, for a Coulomb potential it is of order $h|\log h|$, and for a general finite energy electromagnetic field it is of order $h^{1 / 2}$.
\end{abstract}

\section{Introduction}

The Schrödinger equation can be used to describe for instance electrons in a non-relativistic setting and is therefore of fundamental importance. Recent progress in manipulating such basic systems promises technological breakthroughs such as quantum computing. Electrons are manipulated by magnetic traps, electrostatic potentials and laser beams all of which are electromagnetic fields. When the electromagnetic field is strong it can be described classically by Maxwell's equations. The Schrödinger equation is modified accordingly and involves an electromagnetic gauge potential.

In this paper we introduce and study a numerical method for computing the eigenvalues of the Schrödinger operator in the presence of an electromagnetic field. They correspond to possible energy levels for the electron.

When the electromagnetic field is represented by a gauge potential there is some arbitrariness. Adding a gradient to the magnetic potential and doing a corresponding phase shift on the wave function does not fundamentally change the system (but rather our description of it). Importantly, the energy levels of the electron as well as the associated probability densities, are independent of the choice of gauge. We want to design a numerical method with the same property.

Lattice gauge theory [16] is a discretization technique with such an invariance property. It can be used for the Schrödinger equation [11], and we have previously applied it to the Maxwell-Klein-Gordon equation [8], though it was invented for more complicated systems (quantum fields with non-commutative gauge group). However, to the best of our knowledge, no numerical analysis of this method is available and moreover it is formulated for discretizations of the physical domain using Cartesian grids. In this paper

*snorrec@math.uio.no

†t.g.halvorsen@cma.uio.no 
we modify lattice gauge theory using techniques from finite element methods, such as mass-lumping, to obtain a method formulated on simplicial grids (one consisting of tetrahedrons). Moreover we study the error of the method by comparing it with a standard Galerkin finite element method.

Simplicial meshes are better at handling boundaries of domains which is important for technological applications. When treating singular fields, such as Coulomb potentials, local mesh refinements are also useful and for this reason too simplicial grids might be preferable.

Lattice gauge theory was introduced to handle quantum fields. It is quite possible that the proposed techniques can be used also when the electromagnetic field is treated quantum-mechanically.

We notice that by separating the modulus and the phase of the wave-function it is possible to obtain another gauge invariant discretization [2] allowing for high order finite elements. Due to problems of definition and regularity of the phase where the modulus vanishes this method seems best for computing the fundamental state corresponding to the lowest eigenvalue. Our method can be used for all the eigenvalues but on the other hand it is confined to lowest order finite elements.

We first introduce the mathematical setting for the Schrödinger equation in an electromagnetic field and briefly recall some physical facts. Then we review discretization tools including finite element exterior calculus (mixed finite elements or Whitney forms) and mass-lumping. Then we introduce the proposed discrete eigenvalue problem and state its gauge invariance property. Finally we study the error committed.

\section{The continuous Schrödinger eigenvalue problem}

Let $S \subset \mathbb{R}^{3}$ be a bounded domain in space whose boundary is smooth enough (e.g. locally the graph of Lipschitz functions). We use the standard Euclidean product on $\mathbb{R}^{3}$ through which vector fields will be identified with one-forms or two-forms, scalar fields with zero-forms or three-forms. The real valued $\mathrm{L}^{2}$ product on differential forms on $S$ is denoted $\langle\cdot, \cdot\rangle$, and the associated $\mathrm{L}^{2}$-norm $\|\cdot\|$. If we want to use these norms on some domain $S^{\prime}$ different from $S$ we write $\langle\cdot, \cdot\rangle_{S^{\prime}}$ and $\|\cdot\|_{S^{\prime}}$. For any differential operator op we define the Sobolev spaces:

$$
\mathrm{H}_{\mathrm{op}}(S)=\left\{u \in \mathrm{L}^{2}(S): \text { op } u \in \mathrm{L}^{2}(S)\right\},
$$

where $\mathrm{L}^{2}(S)$ spaces of differential forms or vector and scalar fields are assumed. We then have a diagram of Hilbert spaces linked by bounded operators with closed range:

$$
\mathrm{H}_{\text {grad }}(S) \stackrel{\text { grad }}{\longrightarrow} \mathrm{H}_{\text {curl }}(S) \stackrel{\text { curl }}{\longrightarrow} \mathrm{H}_{\text {div }}(S) \stackrel{\text { div }}{\longrightarrow} \mathrm{H}(S) .
$$

We are given a magnetic field $B$ on $\mathbb{R}^{3}$, which is a closed two-form identified with a divergence free vector field. We assume at least locally finite energy, i.e. $\|B\|_{S^{\prime}}<+\infty$ for any bounded $S^{\prime}$. The magnetic field can be represented by a magnetic potential $A$ on $\mathbb{R}^{3}$ which is also locally $\mathrm{L}^{2}$. It is a vector field or one-form such that $\operatorname{curl} A=B$. We are also given an electric field $E$. We consider only time-constant electromagnetic fields, so $E$ is represented by an electric potential $V$ which is a function on $\mathbb{R}^{3}$ such that $\operatorname{grad} V=E$. The electric potential will be the sum of locally $\mathrm{H}_{\text {grad }}$ functions and Coulomb potentials. The former condition guarantees locally finite energy $\left(\|E\|_{S^{\prime}}<+\infty\right.$ for any bounded $\left.S^{\prime}\right)$ while the latter is also important in applications.

Up until now we have assumed real valued vector fields and differential forms. A wave function is a complex function $\psi$ on $\mathbb{R}^{3}$ which is in $\mathrm{H}_{\text {grad }}\left(S^{\prime}\right) \otimes \mathbb{C}$ for any bounded $S^{\prime}$. The covariant gradient of $\psi$ is:

$$
\operatorname{grad}_{A} \psi=\operatorname{grad} \psi+i A \psi
$$

where grad now acts on complex functions.

For definiteness we shall assume that the domain $S$ is filled with vacuum whereas the rest of $\mathbb{R}^{3}$ is filled with a perfect conductor. The wave function has support in $S$ so that $\psi \in \mathrm{H}_{0}^{1}(S) \otimes \mathbb{C}$.

The Schrödinger eigenvalue problem consists in finding $(\psi, \lambda) \in\left(\mathrm{H}_{0}^{1}(S) \otimes \mathbb{C}\right) \times \mathbb{R}, \psi \neq 0$, which solves the equation:

$$
\forall \psi^{\prime} \in \mathrm{H}_{0}^{1}(S) \otimes \mathbb{C} \quad a\left(\psi, \psi^{\prime}\right)+b\left(\psi, \psi^{\prime}\right)=\lambda\left\langle\psi, \psi^{\prime}\right\rangle,
$$


where $a(\cdot, \cdot)$ and $b(\cdot, \cdot)$ are the bilinear forms given by:

$$
\begin{aligned}
& a\left(\psi, \psi^{\prime}\right)=\left\langle\operatorname{grad}_{A} \psi, \operatorname{grad}_{A} \psi^{\prime}\right\rangle, \\
& b\left(\psi, \psi^{\prime}\right)=\left\langle V \psi, \psi^{\prime}\right\rangle,
\end{aligned}
$$

Most often we will chose the normalization $\|\psi\|=1$. Since it will usually be clear if the fields considered are real or complex we will omit the precision from the notation from now on.

We assume that the electromagnetic field is not affected by the wave function. Notice also that even though the eigenvalue problem is formulated on $S$, the magnetic potential $A$ depends on the values of $B$ outside $S$ and this can have a non-trivial effect on the wave function, as illustrated by the Aharonov-Bohm effect. Even if $B$ is zero on $S$ it may be that no $A$ which is zero on $S$ can represent it on $\mathbb{R}^{3}$.

A fundamental property of equation (4) is that it is invariant under local $\mathbb{U}(1)$-transformations called gauge transformations. They are of the form:

$$
\begin{aligned}
& A \mapsto A^{\prime}=A-\operatorname{grad} \beta, \\
& \psi \mapsto \psi^{\prime}=e^{i \beta} \psi,
\end{aligned}
$$

where $\beta$ is a real scalar field on $\mathbb{R}^{3}$ which is at least locally in $\mathrm{H}_{\text {grad }}$. By invariance it is meant that the transformed gauge potential $A^{\prime}$ represents the same magnetic field $B$ and that if $(\psi, \lambda)$ solves the eigenvalue problem with respect to $A$ then $\left(\psi^{\prime}, \lambda\right)$ solves the eigenvalue problem with respect to $A^{\prime}$. Notice that the field $|\psi|^{2}$, which is interpreted as a probability density, is unchanged by gauge transformations. The electromagnetic field $(E, B)$, the energy $\lambda$ and the probability density $|\psi|^{2}$ are usually thought of as physically measurable quantities whereas gauge invariance shows that there is redundancy in the information contained in $(A, \psi)$.

Proposition 2.1. If $A \in \mathrm{L}^{3}(S)$ then a is continuous and coercive on $\mathrm{H}_{0}^{1}(S)$; there is $C>0$ such that:

$$
\forall \psi \in \mathrm{H}_{0}^{1}(S) \quad a(\psi, \psi) \geq 1 / C\|\psi\|_{\mathrm{H}^{1}(S)}^{2} .
$$

Also if $V \in \mathrm{L}^{3 / 2}(S)$ then $b$ is compact on $\mathrm{H}_{0}^{1}(S)$.

Proof. We write:

$$
a\left(\psi, \psi^{\prime}\right)=z\left(\psi, \psi^{\prime}\right)+k\left(\psi, \psi^{\prime}\right),
$$

with:

$$
\begin{aligned}
& z\left(\psi, \psi^{\prime}\right)=\left\langle\operatorname{grad} \psi, \operatorname{grad} \psi^{\prime}\right\rangle, \\
& k\left(\psi, \psi^{\prime}\right)=\left\langle\operatorname{grad} \psi, i A \psi^{\prime}\right\rangle+\left\langle i A \psi, \operatorname{grad} \psi^{\prime}\right\rangle+\left\langle i A \psi, i A \psi^{\prime}\right\rangle .
\end{aligned}
$$

Observe first that $z$ is coercive on $\mathrm{H}_{0}^{1}(S)$ by the Poincaré inequality.

Second, recall that we have a Sobolev injection $\mathrm{H}^{1}(S) \rightarrow \mathrm{L}^{6}(S)$ and that Hölder's inequality shows the continuity of the product as a bilinear map $\mathrm{L}^{3}(S) \times \mathrm{L}^{6}(S) \rightarrow \mathrm{L}^{2}(S)$. Therefore when $A$ is $\mathrm{L}^{3}(S)$ we have a continuous operator:

$$
\Xi:\left\{\begin{aligned}
\mathrm{H}^{1}(S) & \rightarrow \mathrm{L}^{2}(S), \\
\psi & \mapsto A \psi .
\end{aligned}\right.
$$

For $A \in \mathrm{L}^{\infty}(S), \Xi$ is compact by Rellich compactness $\mathrm{H}^{1}(S) \rightarrow \mathrm{L}^{2}(S)$. Since $\mathrm{L}^{\infty}(S)$ is dense in $\mathrm{L}^{3}(S)$ and the norm limit of compact operators is compact, $\Xi$ is compact also for $A \in \mathrm{L}^{3}(S)$. Therefore $k$ is compact on $\mathrm{H}_{0}^{1}(S)$.

Finally Kato's inequality [12]:

$$
|\operatorname{grad}| \psi|| \leq\left|\operatorname{grad}_{A} \psi\right|,
$$

shows that $a$ is non-degenerate on $\mathrm{H}_{0}^{1}(S)$ and non-negative.

These three properties together imply that $a$ is coercive on $\mathrm{H}_{0}^{1}(S)$.

Compactness of $b$ when $V \in \mathrm{L}^{3 / 2}(S)$ follows from similar arguments (notice that we have $2 / 3+1 / 6+$ $1 / 6=1)$.

From this it follows that the eigenpairs can be ordered in a sequence such that the eigenvalues increase (with possible degeneracies) and diverge and such that the eigenvectors provide an orthonormal basis for $\mathrm{L}^{2}(S)$. If $V \geq 0$ the smallest eigenvalue is (strictly) positive. 


\section{Discretization tools}

We would like to provide a discretization which is not only convergent but also keeps the invariance properties of the continuous equations under gauge-transformations. As has already been remarked, lattice gauge theory provides a finite difference method on Cartesian grids which has a discrete gauge invariance. Our aim is to extend the lattice gauge theory method to unstructured simplicial grids using techniques from finite element methods. We also provide an error analysis of the method by comparing the proposed method with Galerkin methods using Strang's notion of variational crimes.

We will use the framework of finite element exterior calculus [3], a synthesis of Whitney forms [15] and mixed finite elements [14] initiated by Bossavit [6].

We equip $S$ with a simplicial mesh $\mathscr{T}$, so that we have a partition of our domain into tetrahedral with matching faces. The set of $k$-dimensional simplexes in the mesh is denoted $\mathscr{T}^{k}$. All simplexes are equipped with an orientation. If $T$ is a $k$-simplex and $T^{\prime}$ is a $(k-1)$-simplex in its boundary we let $\epsilon\left(T, T^{\prime}\right)= \pm 1$ according to their relative orientation. For simplexes not in this situation we let $\epsilon\left(T, T^{\prime}\right)=0$. For each $k$ we may view $\epsilon$ as a matrix indexed by $\mathscr{T}^{k} \times \mathscr{T}^{k-1}$ called the incidence matrix. A $k$-cochain is a map $\mathscr{T}^{k} \rightarrow \mathbb{R}$; let $C^{k}$ denote the vector space they form. The incidence matrix provides an operator $\delta: C^{k-1} \rightarrow C^{k}$ called the coboundary operator. The cochain spaces, linked by coboundary operators, form a complex in the sense that for each $k, \delta \delta=0$ as a map $C^{k} \rightarrow C^{k+2}$.

Let $X^{k}$ be the space of Whitney $k$-forms on $S$ associated with $\mathscr{T}$. It is constructed as follows. For any vertex $i$ let $\lambda_{i}$ denote the corresponding barycentric coordinate map. It is the continuous piecewise affine map with value 1 at vertex $i$ and value 0 at other vertexes. For $k \geq 1$ and any $k$-simplex $T \in \mathscr{T}^{k}$ define the associated Whitney $k$-form $\lambda_{T}$ by:

$$
\lambda_{T}=k ! \sum_{i=0}^{k}(-1)^{i} \lambda_{i} \mathrm{~d} \lambda_{0} \wedge \cdots\left(\mathrm{d} \lambda_{i}\right)^{\wedge} \cdots \wedge \mathrm{d} \lambda_{k},
$$

where we have numbered the vertices of $T$ from 0 to $k$ in accordance with its orientation (the result is independent of the choice of numbering) and $(\cdot)^{\wedge}$ means that we omit this term. The family of Whitney $k$-forms $\lambda_{T}$ indexed by $T \in \mathscr{T}^{k}$ constitutes a basis for $X^{k}$. For any $u \in X^{k}$ we denote by $[u]$ the coordinate vector of $u$ in this basis. This provides an isomorphism [.] : $X^{k} \rightarrow C^{k}$. Actually we have for each $T \in \mathscr{T}^{k}$ :

$$
[u]_{T}=\int_{T} u .
$$

Moreover $X^{k}$ is a subspace of $\mathrm{H}_{\mathrm{d}}(S)$ and, identifying the exterior derivative $\mathrm{d}$ with grad, curl and div according to the degree of the differential form, we have a commuting diagram:

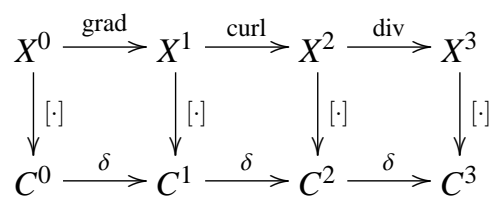

One also defines interpolation operators $I^{k}$, which are projections onto $X^{k}$, by the formula:

$$
I^{k} u=\sum_{T \in \mathscr{T}^{k}}\left(\int_{T} u\right) \lambda_{T}
$$

From Stokes theorem it follows that interpolation commutes with the exterior derivative.

In the following we suppose we have a family of meshes indexed by $h$, and we write for instance $\mathscr{T}_{h}$, $X_{h}^{k}$ and $I_{h}^{k}$ to indicate the dependence upon $h$. The parameter $h$ is also the largest of the diameters of the simplexes of $\mathscr{T}_{h}$. The symbol $C$ appearing in inequalities denotes a numerical constant which may have to be chosen larger in each appearance but is independent of $h$. We suppose that the meshes are quasi-uniform to allow for inverse estimates and that moreover we have for each edge with vertices $i$ and $j$ :

$$
\int \operatorname{grad} \lambda_{i} \cdot \operatorname{grad} \lambda_{j} \leq 0
$$


This condition has previously appeared in connection with discrete maximum principles [10].

The mass matrix $M_{h}^{k}$ is the matrix of the $\mathrm{L}^{2}$ scalar product $\langle\cdot, \cdot\rangle$ in the basis of Whitney $k$-forms. The matrix $M_{h}^{k}$ indexed by $\mathscr{T}_{h}^{k} \times \mathscr{T}_{h}^{k}$ is sparse since the value $\left(M_{h}^{k}\right)_{T T^{\prime}}$ can be nonzero only if $T$ and $T^{\prime}$ are in a common tetrahedron. However it is in general non-diagonal. A procedure yielding a diagonal matrix which is a good approximation of $M_{h}^{k}$ is called mass-lumping. The reason we are interested in mass-lumping is that the locality of gauge transformations conflicts with the type of nearest neighbour interactions present in the mass matrices. For $k=0$ it is well-known that the diagonal matrix $\tilde{M}_{h}^{0}$ defined by:

$$
\left(\tilde{M}_{h}^{0}\right)_{i i}=\sum_{j}\left(M_{h}^{0}\right)_{i j}
$$

is a good mass-lumped version of $M_{h}^{0}$. However mass-lumping $M_{h}^{1}$ is a much more difficult problem for which we know of no solution satisfactory for all purposes. But we do have the following [7]:

Proposition 3.1. There is a unique diagonal matrix $\tilde{M}_{h}^{1}$ such that $\delta^{\mathrm{T}}\left(M_{h}^{1}-\tilde{M}_{h}^{1}\right) \delta=0$.

Ideally the mass lumped matrix should be exact at least on piecewise constant vector fields. In particular it should be exact on gradients of elements in $X_{h}^{0}$. The above proposition states that this uniquely determines $\tilde{M}_{h}^{1}$. Several remarks are in order. First, if an edge $e$ has vertices $i, j$ we have:

$$
\left(\tilde{M}_{h}^{1}\right)_{e e}=-\left\langle\operatorname{grad} \lambda_{i}, \operatorname{grad} \lambda_{j}\right\rangle .
$$

Second, $\tilde{M}_{h}^{1}$ can be constructed from contributions of each tetrahedron which are also diagonal. We can write:

$$
\begin{aligned}
\tilde{M}_{h}^{1} & =\sum_{T \in \mathscr{T}_{h}^{3}} \tilde{M}^{1}(T), \\
\tilde{M}^{1}(T)_{e e} & =-\int_{T} \operatorname{grad} \lambda_{i} \cdot \operatorname{grad} \lambda_{j}, \quad e=\{i, j\} .
\end{aligned}
$$

and the contribution from tetrahedron $T$ is exact when the fields are constant on it.

Third we have uniform boundedness:

$$
\left|\tilde{M}_{h}^{1}[u] \cdot[v]\right| \leq C\|u\|\|v\|,
$$

and this estimate can be derived from an analogous result local to each tetrahedron.

The reason this construction is not satisfactory for all purposes is that on distorted meshes $\tilde{M}_{h}^{1}$ may have negative entries. Even on nice structured meshes such as the barycentric refinement of a cubical lattice, $\tilde{M}_{h}^{1}$ will have zeroes on the diagonal, in breach of the requirement that a mass matrix should be positive definite. Usually uniform positive definiteness is necessary to obtain a stable numerical method.

However we shall need to apply the lumped mass-matrix only to covariant gradients. Since covariant gradients resemble gradients and the lumped mass-matrix is exact for these, there is hope that the method will work. The following estimate improves that of [7].

Proposition 3.2. There exists a constant $C$ such that for all $h$ and all $u, v \in X_{h}^{1}$,

$$
\left|\left(M_{h}^{1}-\tilde{M}_{h}^{1}\right)[u] \cdot[v]\right| \leq C h(\|u\|\|\operatorname{curl} v\|+\|\operatorname{curl} u\|\|v\|) .
$$

Proof. We will consider that the elements of $X_{h}^{1}$ are vector fields and use the notations of Nédélec's edge elements. For any tetrahedron $T$ denote by $x_{T}$ its barycentre. For $u, v \in X_{h}^{1}$ there exists for each tetrahedron $T$, unique vectors $a_{T}, b_{T}, c_{T}$ and $d_{T}$ such that on it:

$$
u(x)=a_{T} \times\left(x-x_{T}\right)+b_{T}, \quad v(x)=c_{T} \times\left(x-x_{T}\right)+d_{T} .
$$

Observe that $a_{T}=1 / 2 \operatorname{curl} u$ and $c_{T}=1 / 2 \operatorname{curl} v$. Also, by the choice of origin, $b_{T}$ and $d_{T}$ are the $\mathrm{L}^{2}(S)$ projections of $u$ and $v$ on the space of constant vector fields. 
Since on $T$ we have $\left|x-x_{T}\right| \leq h$ :

$$
\begin{aligned}
\left|\left(M^{1}(T)-\tilde{M}^{1}(T)\right)\left[u-b_{T}\right] \cdot[v]\right| & \leq C\left\|u-b_{T}\right\|_{T}\|v\|_{T} \leq C h\|\operatorname{curl} u\|_{T}\|v\|_{T}, \\
\left|\left(M^{1}(T)-\tilde{M}^{1}(T)\right)\left[b_{T}\right] \cdot\left[v-d_{T}\right]\right| & \leq C\left\|b_{T}\right\|_{T}\left\|v-d_{T}\right\|_{T} \leq C h\|u\|_{T}\|\operatorname{curl} v\|_{T}, \\
\left|\left(M^{1}(T)-\tilde{M}^{1}(T)\right)\left[b_{T}\right] \cdot\left[d_{T}\right]\right| & =0 .
\end{aligned}
$$

Adding the three estimates we get:

$$
\left|\left(M^{1}(T)-\tilde{M}^{1}(T)\right)[u] \cdot[v]\right| \leq C h\left(\|u\|_{T}\|\operatorname{curl} v\|_{T}+\|\operatorname{curl} u\|_{T}\|v\|_{T}\right) .
$$

Summing with respect to $T$ and the Cauchy-Schwartz inequality give the desired result.

A similar proof shows (when mass lumping $M_{h}^{0}$ as indicated in (20), it is enough that one of the fields $u, v \in X_{h}^{0}$ is constant on $T$ for the contribution of $\tilde{M}^{0}(T)$ to be exact on it):

Proposition 3.3. There exists a constant $C$ such that for all $h$ and all $u, v \in X_{h}^{0}$,

$$
\begin{aligned}
& \left|\left(M_{h}^{0}-\tilde{M}_{h}^{0}\right)[u] \cdot[v]\right| \leq C h\|u\|\|\operatorname{grad} v\|, \\
& \left|\left(M_{h}^{0}-\tilde{M}_{h}^{0}\right)[u] \cdot[v]\right| \leq C h^{2}\|\operatorname{grad} u\|\|\operatorname{grad} v\| .
\end{aligned}
$$

We also have the well known estimates for $u \in X_{h}^{0}$ :

$$
1 / C\|u\|^{2} \leq \tilde{M}_{h}^{0}[u] \cdot[u] \leq C\|u\|^{2} .
$$

As already remarked the right hand inequality has an analogue for $\tilde{M}_{h}^{1}$ but not the left hand one.

The above estimates can also be obtained by scaling arguments. Consider a so-called reference tetrahedron $\hat{T}$ of diameter 1 and a tetrahedron $T$ of diameter $h$ obtained from $\hat{T}$ by the a map $\Phi: x \rightarrow h x$ (perhaps composed with a congruence). Let $u$ be a $k$-form on $T$ and $\hat{u}=\Phi^{\star} u$ the pull-back of $u$ to $\hat{T}$. Then we have:

$$
\|u\|_{L^{p}(T)}=h^{-k+n / p}\|\hat{u}\|_{L^{p}(\hat{T})},
$$

where $n$ is the dimension of the ambient space, so that $n=3$ in our case.

From this one can deduce inverse estimates and convergence estimates. In particular we will use that for $u \in X_{h}^{k}$ :

$$
\|u\|_{\mathrm{L}^{3}(S)} \leq C h^{-1 / 2}\|u\|_{\mathrm{L}^{2}(S)} .
$$

For projectors $\Pi_{h}^{k}$ onto $X_{h}^{k}$ which have the property that the values of $\Pi_{h}^{k} u$ on tetrahedron $T \in \mathscr{T}_{h}$ depend continuously on the values of $u$ on the macro-element $\bar{T}$ of $T$ (the union of tetrahedrons intersecting $T$ ) with respect to the $\mathrm{L}^{2}(\bar{T})$ norm and which commute with scaling on macro elements, on obtains estimates for $k$-forms $u$ in $\mathrm{H}^{1}(S)$ :

$$
\left\|u-\Pi_{h}^{k} u\right\|_{\mathrm{L}^{2}(S)} \leq C h\|u\|_{\mathrm{H}^{1}(S)} .
$$

\section{The discrete eigenvalue problem}

We introduce the spaces $Y_{h}^{k}=X_{h}^{k} \otimes \mathbb{C}$. The Galerkin discretization of the eigenvalue problem is to find $(u, \lambda)$ in $Y_{h}^{0} \times \mathbb{R}$ such that:

$$
\forall v \in Y_{h}^{0} \quad a(u, v)+b(u, v)=\lambda\langle u, v\rangle .
$$

This is a well studied problem with quasi-optimal convergence [4]. Our contribution is to propose and study a modification of it which exhibits a form of gauge invariance.

Choose $A_{h} \in X_{h}^{1}$ such that $\left(A_{h}\right)$ converges to $A$ in a sense which will be made precise in the next section. We define the symmetric bilinear form $a_{h}$ as follows. For any oriented edge $e=\{i, j\} \in \mathscr{T}_{h}^{1}$ denote:

$$
\mu_{i j}^{h}=\left(\tilde{M}_{h}^{1}\right)_{e e}, \quad\left[A_{h}\right]_{i j}=\left[A_{h}\right]_{e}=\int_{e} A_{h}
$$


We have $\mu_{i j}^{h} \geq 0$ by hypothesis (19). Define for $u \in Y_{h}^{0}$ :

$$
a_{h}(u, u)=\sum_{\{i, j\} \in \mathscr{T}_{h}^{1}} \mu_{i j}^{h}\left|u_{j}-\exp \left(-i\left[A_{h}\right]_{i j}\right) u_{i}\right|^{2} .
$$

We also choose $V_{h} \in X_{h}^{0}$ such that $\left(V_{h}\right)$ converges to $V$ in appropriate norms. For a vertex $i \in \mathscr{T}_{h}^{0}$ denote:

$$
\mu_{i}^{h}=\left(\tilde{M}_{h}^{0}\right)_{i i}, \quad\left[V_{h}\right]_{i}=V_{h}(i) .
$$

For $u \in Y_{h}^{0}$ define:

$$
b_{h}(u, u)=\sum_{i \in \mathscr{T}_{h}^{0}} \mu_{i}\left[V_{h}\right]_{i}\left|u_{i}\right|^{2}
$$

We will also use the notation:

$$
\langle u, u\rangle_{h}=\tilde{M}_{h}^{0}[u] \cdot[u] .
$$

We propose to solve: find $(u, \lambda)$ in $Y_{h}^{0} \times \mathbb{R}, u \neq 0$ such that:

$$
\forall v \in Y_{h}^{0} \quad a_{h}(u, v)+b_{h}(u, v)=\lambda\langle u, v\rangle_{h} .
$$

Before we study the error in this method we exhibit the discrete gauge invariance which motivated the use of lattice gauge theory.

Given $\beta_{h} \in X_{h}^{0}$ we consider the transformations:

$$
\begin{gathered}
X_{h}^{1} \rightarrow X_{h}^{1}: A_{h} \mapsto A_{h}^{\prime}=A_{h}-\operatorname{grad} \beta_{h}, \\
Y_{h}^{0} \rightarrow Y_{h}^{0}: u \mapsto u^{\prime}=I_{h}^{0}\left(\exp \left(i \beta_{h}\right) u\right) .
\end{gathered}
$$

Theorem 4.1. The discrete eigenvectors computed by (43) using $A_{h}^{\prime}$ are obtained from those computed using $A_{h}$ by the transformation (45). The discrete eigenvalues are unchanged and for any eigenvector $u$ the field:

$$
p_{h}=\sum_{i \in \mathscr{T}_{h}^{0}}\left|u_{i}\right|^{2} \lambda_{i} \in X_{h}^{0}
$$

is gauge independent and plays the role of a probability density.

Proof. Let $a_{h}^{\prime}$ be the bilinear form obtained from $a_{h}$ by replacing $A_{h}$ with $A_{h}^{\prime}$ in (39). With the above notations $(44,45)$ we have:

$$
a_{h}^{\prime}\left(u^{\prime}, u^{\prime}\right)=a_{h}(u, u), \quad b_{h}\left(u^{\prime}, u^{\prime}\right)=b_{h}(u, u), \quad\left\langle u^{\prime}, u^{\prime}\right\rangle_{h}=\langle u, u\rangle_{h},
$$

from which the result follows by the characterization of eigenpairs in terms of Rayleigh quotients.

Notice that this is not achieved by the standard Galerkin method (37). If the nodal interpolator is not included in (45) one is mapped out of the Galerkin space, but if it is included, the expression $a(u, u)$ is not gauge invariant.

There are other interesting gauge invariant quantities, such as the flux:

$$
f_{h}=\sum_{\{i, j\} \in \mathscr{T}_{h}^{1}} \mathfrak{I}\left(u_{j}^{\star} \exp \left(-i\left[A_{h}\right]_{i j}\right) u_{i}\right) \lambda_{i j} \in X_{h}^{1} .
$$

\section{Error analysis}

The minimal regularity we shall assume for the gauge potential $A$ is $\mathrm{H}^{1}(S)$ but we will also derive error estimates for the case when $A$ is smooth. When $B$ is locally $\mathrm{L}^{2}$, choosing a divergence-free $A$ on $\mathbb{R}^{3}$ will give $\mathrm{H}^{1}(S)$ regularity for $A$, but we do not confine our study to this gauge. Sobolev spaces are reviewed in [1] and classical error estimates for mixed finite elements can be found in [13]. 
Clément style interpolation [5] will yield convergence estimates:

$$
\left\|A-A_{h}\right\|_{\mathrm{L}^{3}(S)} \leq C h^{1 / 2}\|A\|_{\mathrm{H}^{1}(S)} .
$$

The $\mathrm{L}^{p}$ stable interpolators commuting with the exterior derivative introduced in [9] will achieve the same order of convergence and in addition bounds:

$$
\begin{gathered}
\left\|A_{h}\right\|_{\mathrm{L}^{6}(S)} \leq C\|A\|_{\mathrm{L}^{6}(S)}, \\
\left\|\operatorname{curl} A_{h}\right\|_{\mathrm{L}^{2}(S)} \leq C\|\operatorname{curl} A\|_{\mathrm{L}^{2}(S)} .
\end{gathered}
$$

If $A$ is smooth $A_{h}=I_{h}^{1} A$ will achieve:

$$
\begin{aligned}
\left\|A-A_{h}\right\|_{\mathrm{L}^{\infty}(S)} & \leq C h\|A\|_{\mathrm{W}^{1, \infty}(S)}, \\
\left\|A_{h}\right\|_{\mathrm{L}^{\infty}(S)} & \leq C\|A\|_{\mathrm{L}^{\infty}(S)}, \\
\left\|\operatorname{curl} A_{h}\right\|_{\mathrm{L}^{\infty}(S)} & \leq C\|\operatorname{curl} A\|_{\mathrm{L}^{\infty}(S)} .
\end{aligned}
$$

Coulomb potentials are in $\mathrm{W}^{1,3 \delta / 2}(S)$ for $\delta<1$. Generally if $V \in \mathrm{W}^{1,3 \delta / 2}(S)$ with $\delta \leq 1$ we can similarly achieve:

$$
\begin{gathered}
\left\|V-V_{h}\right\|_{\mathrm{L}^{3 \delta / 2}(S)} \leq C h\|\operatorname{grad} V\|_{\mathrm{L}^{3 \delta / 2}}, \\
\left\|V_{h}\right\|_{\mathrm{W}^{1,3 \delta / 2}(S)} \leq C\|V\|_{\mathrm{W}^{1,3 \delta / 2}(S)} .
\end{gathered}
$$

This hypothesis includes all finite energy electric fields. Additional smoothness of $V$ does not seem to improve the error estimates we shall obtain below.

To justify the proposed method we introduce three other bilinear forms providing intermediate steps between $a$ and $a_{h}$. Define:

$$
a_{h}^{0}(u, v)=\left\langle\operatorname{grad}_{A_{h}} u, \operatorname{grad}_{A_{h}} v\right\rangle .
$$

The product $A_{h} u$ is not in $Y_{h}^{1}$ but interpolating it down we define:

$$
a_{h}^{1}(u, v)=\left\langle\operatorname{grad} u+I_{h}^{1}\left(i A_{h} u\right), \operatorname{grad} v+I_{h}^{1}\left(i A_{h} v\right)\right\rangle .
$$

Next we use mass-lumped matrices:

$$
a_{h}^{2}(u, v)=\tilde{M}_{h}^{1}\left[\operatorname{grad} v+I_{h}^{1}\left(i A_{h} v\right)\right] \cdot\left[\operatorname{grad} u+I_{h}^{1}\left(i A_{h} u\right)\right] .
$$

We shall evaluate the errors for each step of approximation:

$$
a \rightarrow a_{h}^{0} \rightarrow a_{h}^{1} \rightarrow a_{h}^{2} \rightarrow a_{h}
$$

First concerning the use of an approximate gauge field we have:

Proposition 5.1. We have:

$$
\left|a(u, v)-a_{h}^{0}(u, v)\right| \leq C h^{1 / 2}\|u\|_{\mathrm{H}^{1}(S)}\|v\|_{\mathrm{H}^{1}(S)} .
$$

Proof. It follows from (49).

Remark 5.1. If $A$ is smooth we get:

$$
\left|a(u, v)-a_{h}^{0}(u, v)\right| \leq C h\|u\|_{\mathrm{H}^{1}(S)}\|v\|_{\mathrm{H}^{1}(S)} .
$$

Second the interpolation of the product contributes the following error:

Proposition 5.2. We have:

$$
\left|a_{h}^{0}(u, v)-a_{h}^{1}(u, v)\right| \leq C h^{1 / 2}\|u\|_{\mathrm{H}^{1}(S)}\|v\|_{\mathrm{H}^{1}(S)} .
$$


Proof. Given $A_{h} \in X_{h}^{1}$ and $u \in Y_{h}^{0}$ we interpolate their product to obtain a field in $Y_{h}^{1}$.

Remark that if $u$ is constant on a given tetrahedron there is no error committed there - the interpolation is exact. On a reference tetrahedron $\hat{T}$ we therefore have an estimate of the form:

$$
\left\|A_{h} u-I_{h}^{1}\left(A_{h} u\right)\right\|_{L^{2}(\hat{T})} \leq C\left\|A_{h}\right\|_{L^{6}(\hat{T})}\|\operatorname{grad} u\|_{L^{3}(\hat{T})} .
$$

Squaring and scaling to a tetrahedron $T$ of size $h$ gives:

$$
\left\|A_{h} u-I_{h}^{1}\left(A_{h} u\right)\right\|_{\mathrm{L}^{2}(T)}^{2} \leq C h^{2}\left\|A_{h}\right\|_{\mathrm{L}^{6}(T)}^{2}\|\operatorname{grad} u\|_{\mathrm{L}^{3}(T)}^{2} .
$$

Summing over tetrahedrons and using Hölder's inequality gives:

$$
\left\|A_{h} u-I_{h}^{1}\left(A_{h} u\right)\right\|_{\mathrm{L}^{2}(S)}^{2} \leq C h^{2}\left\|A_{h}\right\|_{\mathrm{L}^{6}(S)}^{2}\|\operatorname{grad} u\|_{\mathrm{L}^{3}(S)}^{2} .
$$

A square-root and inverse inequality gives:

$$
\left\|A_{h} u-I_{h}^{1}\left(A_{h} u\right)\right\|_{\mathrm{L}^{2}(S)} \leq C h^{1 / 2}\left\|A_{h}\right\|_{\mathrm{L}^{6}(S)}\|\operatorname{grad} u\|_{\mathrm{L}^{2}(S)} .
$$

From this the proposition follows.

Remark 5.2. If $\mathrm{A}$ is smooth we can replace $\mathrm{L}^{6} \times \mathrm{L}^{3} \rightarrow \mathrm{L}^{2}$ by $\mathrm{L}^{\infty} \times \mathrm{L}^{2} \rightarrow \mathrm{L}^{2}$, and there is no need for an inverse inequality, so we get:

$$
\left|a_{h}^{0}(u, v)-a_{h}^{1}(u, v)\right| \leq C h\|u\|_{\mathrm{H}^{1}(S)}\|v\|_{\mathrm{H}^{1}(S)} .
$$

Third, mass-lumping induces the following error:

Proposition 5.3. We have:

$$
\left|a_{h}^{1}(u, v)-a_{h}^{2}(u, v)\right| \leq C h^{1 / 2}\|u\|_{\mathrm{H}^{1}(S)}\|v\|_{\mathrm{H}^{1}(S)} .
$$

Proof. Using previous estimates we get:

$$
\left|a_{h}^{1}(u, v)-a_{h}^{2}(u, v)\right| \leq C h\left(\left\|\operatorname{grad} u+I_{h}^{1}\left(i A_{h} u\right)\right\|\left\|\operatorname{curl} I_{h}^{1}\left(A_{h} v\right)\right\|+\left\|\operatorname{curl} I_{h}^{1}\left(A_{h} u\right)\right\|\left\|\operatorname{grad} v+I_{h}^{1}\left(i A_{h} v\right)\right\|\right)
$$

Next we use the uniform stability of interpolation $I_{h}^{1}: Y_{h}^{0} Y_{h}^{1} \rightarrow Y_{h}^{1}$ with respect to $\mathrm{L}^{2}(S) \rightarrow \mathrm{L}^{2}(S)$ norms, Hölder's inequality and Sobolev injections.

$$
\begin{aligned}
\left\|I_{h}^{1}\left(A_{h} u\right)\right\| & \leq C\left\|A_{h} u\right\|, \\
& \leq C\left\|A_{h}\right\|_{\mathrm{L}^{3}(S)}\|u\|_{\mathrm{L}^{6}(S)}, \\
& \leq C\left\|A_{h}\right\|_{\mathrm{L}^{3}(S)}\|u\|_{\mathrm{H}^{1}(S)} .
\end{aligned}
$$

Remark that $Y_{h}^{0} Y_{h}^{k}$ is a finite element space, the one of second lowest order in Nédélec's first family. Therefore curl maps $Y_{h}^{0} Y_{h}^{1} \rightarrow Y_{h}^{0} Y_{h}^{2}$. Moreover $I_{h}^{2}: Y_{h}^{0} Y_{h}^{2} \rightarrow Y_{h}^{2}$ is stable $\mathrm{L}^{2}(S) \rightarrow \mathrm{L}^{2}(S)$ by scaling:

$$
\begin{aligned}
\left\|\operatorname{curl} I_{h}^{1}\left(A_{h} v\right)\right\| & =\left\|I_{h}^{2} \operatorname{curl}\left(A_{h} v\right)\right\|, \\
& \leq C\left\|\operatorname{curl}\left(A_{h} v\right)\right\|, \\
& \leq C\left(\left\|\left(\operatorname{curl} A_{h}\right) v\right\|+\left\|A_{h} \times \operatorname{grad} v\right\|\right), \\
& \leq C\left(\left\|\operatorname{curl} A_{h}\right\|_{\mathrm{L}^{3}(S)}\|v\|_{\mathrm{L}^{6}(S)}+\left\|A_{h}\right\|_{\mathrm{L}^{6}(S)}\|\operatorname{grad} v\|_{\mathrm{L}^{3}(S)}\right), \\
& \leq C h^{-1 / 2}\left(\left\|A_{h}\right\|_{\mathrm{L}^{6}(S)}+\left\|\operatorname{curl} A_{h}\right\|_{\mathrm{L}^{2}(S)}\right)\|v\|_{\mathrm{H}^{1}(S)}
\end{aligned}
$$

where the $h^{-1 / 2}$ factor is obtained from an inverse inequality.

Similar estimates hold with $u$ and $v$ interchanged. Combining them yields the proposition.

Remark 5.3. If $A$ is smooth we get for the same reason as previously:

$$
\left|a_{h}^{1}(u, v)-a_{h}^{2}(u, v)\right| \leq C h\|u\|_{\mathrm{H}^{1}(S)}\|v\|_{\mathrm{H}^{1}(S)} .
$$


Fourth, applying the technique of lattice gauge theory we get:

Proposition 5.4. We have:

$$
\left|a_{h}^{2}(u, v)-a_{h}(u, v)\right| \leq C h^{1 / 2}\|u\|_{\mathrm{H}^{1}(S)}\|v\|_{\mathrm{H}^{1}(S)} .
$$

Proof. Define a function $r: \mathbb{R} \rightarrow \mathbb{C}$ by, for all $\theta \in \mathbb{R}$ :

$$
\exp (i \theta)=1+i \theta-\frac{1}{2} \theta^{2}+r(\theta),
$$

and remark that we have a bound valid for all $\theta \in \mathbb{R}$ :

$$
|r(\theta)| \leq C|\theta|^{3} .
$$

We look at an edge with vertices $i$ and $j$. Put $\theta_{i j}=\left[A_{h}\right]_{i j}$. We have:

$$
\begin{aligned}
\left|u_{j}-\exp \left(-i \theta_{i j}\right) u_{i}\right| & =\left|\exp \left(\frac{i}{2} \theta_{i j}\right) u_{j}-\exp \left(-\frac{i}{2} \theta_{i j}\right) u_{i}\right| \\
& =\left|u_{j}-u_{i}+\frac{i}{2} \theta_{i j}\left(u_{i}+u_{j}\right)-\frac{1}{8} \theta_{i j}^{2}\left(u_{j}-u_{i}\right)+r\left(\frac{1}{2} \theta_{i j}\right) u_{j}-r\left(-\frac{1}{2} \theta_{i j}\right) u_{i}\right| .
\end{aligned}
$$

On the right hand side we recognize:

$$
u_{j}-u_{i}+\frac{i}{2} \theta_{i j}\left(u_{i}+u_{j}\right)=\left[\operatorname{grad} u+I_{h}^{1}\left(i A_{h} u\right)\right]_{i j},
$$

which appears in the expression for $a_{h}^{2}(u, u)$. We want to bound the contribution of the other terms. From the boundedness of $\left(A_{h}\right)$ in $\mathrm{L}^{6}(S)$ we deduce a bound valid for all $h$ and all edges:

$$
\left|\theta_{i j}\right| \leq C h^{1 / 2} .
$$

From which we deduce,

$$
\sum_{i j} \mu_{i j}\left|\theta_{i j}^{2}\left(u_{j}-u_{i}\right)\right|^{2} \leq C h^{2}\|\operatorname{grad} u\|^{2},
$$

and also:

$$
\sum_{i j} \mu_{i j}\left|r\left(\frac{1}{2} \theta_{i j}\right) u_{j}\right|^{2} \leq C h\|u\|^{2} .
$$

This ends the proof.

Remark 5.4. If $A$ is smooth we have a bound $\left|\theta_{i j}\right| \leq C h$, which gives:

$$
\left|a_{h}^{2}(u, v)-a_{h}(u, v)\right| \leq C h^{2}\|u\|_{\mathrm{H}^{1}(S)}\|v\|_{\mathrm{H}^{1}(S)} .
$$

Combining the four estimates gives:

Theorem 5.5. If $A \in \mathrm{H}^{1}(S)$ we have:

$$
\left|a(u, v)-a_{h}(u, v)\right| \leq C h^{1 / 2}\|u\|_{\mathrm{H}^{1}(S)}\|v\|_{\mathrm{H}^{1}(S)},
$$

and if $A$ is smooth:

$$
\left|a(u, v)-a_{h}(u, v)\right| \leq C h\|u\|_{\mathrm{H}^{1}(S)}\|v\|_{\mathrm{H}^{1}(S)} .
$$

It can also be remarked that if $A_{h}=A=0, a_{h}$ is the exact restriction of $a$ to $Y_{h}^{0}$.

Concerning the approximation of $b$ we remark that:

$$
b_{h}(u, u)=\int I_{h}^{0}\left(V_{h}|u|^{2}\right) .
$$


Proposition 5.6. There is $C>0$ such that for all $\delta \leq 1$ we have for all $h$ and all $u \in Y_{h}^{0}$ :

$$
\left|\langle V u, u\rangle-\left\langle V_{h} u, u\right\rangle\right| \leq C h^{2-2 / \delta}\left\|V-V_{h}\right\|_{\mathrm{L}^{3 \delta / 2}(S)}\|u\|_{\mathrm{H}^{1}(S)}^{2} .
$$

Proof. Given $\delta$ define $\delta^{\prime}$ by $2 /(3 \delta)+1 /\left(3 \delta^{\prime}\right)=1$. From Hölder, an inverse inequality and the Sobolev injection $\mathrm{H}^{1}(S) \rightarrow \mathrm{L}^{6}(S)$ we have:

$$
\begin{aligned}
\left|\langle V u, u\rangle-\left\langle V_{h} u, u\right\rangle\right| & \leq\left\|V-V_{h}\right\|_{\mathrm{L}^{3 \delta / 2}}\|u\|_{\mathrm{L}^{6 \delta^{\prime}}}^{2}, \\
& \leq C\left\|V-V_{h}\right\|_{\mathrm{L}^{3 \delta / 2}(S)} h^{1 / \delta^{\prime}-1}\|u\|_{\mathrm{L}^{6}}^{2}, \\
& \leq C h^{2-2 / \delta}\left\|V-V_{h}\right\|_{\mathrm{L}^{3 \delta / 2}(S)}\|u\|_{\mathrm{H}^{1}(S)}^{2} .
\end{aligned}
$$

This is the claimed estimate.

Proposition 5.7. We have for $u \in Y_{h}^{0}$ :

$$
\left.\left|\int V_{h}\right| u\right|^{2}-\int I_{h}^{0}\left(V_{h}|u|^{2}\right) \mid \leq C h\left\|V_{h}\right\|_{\mathrm{W}^{1,3 / 2}(S)}\|u\|_{\mathrm{H}^{1}(S)}^{2} .
$$

Proof. Consider a simplex $T$. Let $V_{h}^{\prime}$ be the $\mathrm{L}^{2}(T)$ projection of $V_{h}$ to the constants. Similarly let $u^{\prime}$ be the $\mathrm{L}^{2}(T)$ projection of $u$ to the constants. We have on the simplex $T$ :

$$
\begin{aligned}
\left(\mathrm{id}-I_{h}^{0}\right)\left(V_{h}|u|^{2}\right) & =\left(\mathrm{id}-I_{h}^{0}\right)\left(V_{h}^{\prime}|u|^{2}\right)+\left(\mathrm{id}-I_{h}^{0}\right)\left(\left(V_{h}-V_{h}^{\prime}\right)|u|^{2}\right), \\
& =\left(\mathrm{id}-I_{h}^{0}\right)\left(V_{h}^{\prime}\left|u-u^{\prime}\right|^{2}\right)+\left(\mathrm{id}-I_{h}^{0}\right)\left(\left(V_{h}-V_{h}^{\prime}\right)|u|^{2}\right) .
\end{aligned}
$$

Therefore:

$$
\left|\int_{T}\left(\operatorname{id}-I_{h}^{0}\right)\left(V_{h}|u|^{2}\right)\right| \leq C h^{2}\left\|V_{h}\right\|_{L^{3}(T)}\|\operatorname{grad} u\|_{L^{3}(T)}^{2}+C h\left\|\operatorname{grad} V_{h}\right\|_{L^{3 / 2}(T)}\|u\|_{L^{6}(T)}^{2} .
$$

We sum over $T$, use discrete Hölder (for $1 / 3+1 / 3+1 / 3=1$ and $2 / 3+1 / 6+1 / 6=1$ ) and the Sobolev injection $W^{1,3 / 2} \rightarrow \mathrm{L}^{3}$. We lose an $h$ in the first term from an inverse inequality.

Theorem 5.8. If $V \in \mathrm{W}^{1,3 / 2}(S)$ we have an estimate for $u, v \in Y_{h}^{0}$ :

$$
\left|b(u, v)-b_{h}(u, v)\right| \leq C h\|u\|_{\mathrm{H}^{1}(S)}\|v\|_{\mathrm{H}^{1}(S)} .
$$

If $V$ is a Coulomb potential we have:

$$
\left|b(u, v)-b_{h}(u, v)\right| \leq C h|\log h|\|u\|_{\mathrm{H}^{1}(S)}\|v\|_{\mathrm{H}^{1}(S)} .
$$

Proof. We treat the case of a Coulomb potential located at the origin, which is supposed to be in the interior of $S$. There is $C>0$ such that for all $\delta<1$ we have:

$$
\left\|1 /|x|^{2}\right\|_{L^{3 \delta / 2}(S)} \leq C /(1-\delta) .
$$

Then :

$$
h^{2-2 / \delta}\left\|V-V_{h}\right\|_{\mathrm{L}^{3 \delta / 2}(S)} \leq C h^{3-2 / \delta} /(1-\delta) .
$$

The choice $1-\delta=-1 / \log h$ leads to the claimed estimate.

\section{Acknowledgements}

This work, conducted as part of the award "Numerical analysis and simulations of geometric wave equations" made under the European Heads of Research Councils and European Science Foundation EURYI (European Young Investigator) Awards scheme, was supported by funds from the Participating Organizations of EURYI and the EC Sixth Framework Program. 


\section{References}

[1] Robert A. Adams and John J. F. Fournier. Sobolev spaces, volume 140 of Pure and Applied Mathematics (Amsterdam). Elsevier/Academic Press, Amsterdam, second edition, 2003.

[2] François Alouges and Virginie Bonnaillie-Noël. Numerical computations of fundamental eigenstates for the Schrödinger operator under constant magnetic field. Numer. Methods Partial Differential Equations, 22(5):1090-1105, 2006.

[3] D. N. Arnold, R. S. Falk, and R. Winther. Finite element exterior calculus, homological techniques, and applications. Acta Numer., 15:1-155, 2006.

[4] I. Babuška and J. Osborn. Eigenvalue problems. In Handbook of numerical analysis, Vol. II, Handb. Numer. Anal., II, pages 641-787. North-Holland, Amsterdam, 1991.

[5] Christine Bernardi and Frédéric Hecht. Quelques propriétés d'approximation des éléments finis de Nédélec, application à l'analyse a posteriori. C. R. Math. Acad. Sci. Paris, 344(7):461-466, 2007.

[6] A. Bossavit. Mixed finite elements and the complex of Whitney forms. In The mathematics of finite elements and applications, VI (Uxbridge, 1987), pages 137-144. Academic Press, London, 1988.

[7] A. Bossavit and L. Kettunen. Yee-like schemes on a tetrahedral mesh, with diagonal lumping. Int. J. Numer. Model.-Electron. Netw. Device Fields, 12(1-2):129-142, Jan-Apr 1999.

[8] S. H. Christiansen and Halvorsen T.G. Solving the Maxwell-Klein-Gordon equation in the lattice gauge theory formalism. Department of Mathematics, University of Oslo, E-print, 7, 2008.

[9] Snorre H. Christiansen and Ragnar Winther. Smoothed projections in finite element exterior calculus. Math. Comp., 77(262):813-829, 2008.

[10] P. G. Ciarlet and P.-A. Raviart. Maximum principle and uniform convergence for the finite element method. Comput. Methods Appl. Mech. Engrg., 2:17-31, 1973.

[11] Michele Governale and Carlo Ungarelli. Gauge-invariant grid discretization of the Schrödinger equation. Phys. Rev. B, 58(12):7816-7821, Sep 1998.

[12] E. H. Lieb and M. Loss. Analysis, volume 14 of Graduate Studies in Mathematics. American Mathematical Society, Providence, RI, second edition, 2001.

[13] P. Monk. Finite element methods for Maxwell's equations. Numerical Mathematics and Scientific Computation. Oxford University Press, New York, 2003.

[14] J.-C. Nédélec. Mixed finite elements in $\mathbf{R}^{3}$. Numer. Math., 35(3):315-341, 1980.

[15] H. Whitney. Geometric integration theory. Princeton University Press, Princeton, N. J., 1957.

[16] K. G. Wilson. Confinement of quarks. Phys. Rev. D, 10(8):2445-2459, 1974. 